\title{
MJN EFFECT OF EDUCATIONAL PROGRAM ON NURSES' PERFORMANCE FOR CHILDREN WITH IDIOPATHIC THROMBOCYTOPENIC PURPURA
}

\author{
Sahar Wasfy ${ }^{1 *}$, Rahma Soliman², Ebtisam El-Sayed ${ }^{3}$, Basma Mahmoud ${ }^{4}$ \\ Pediatric Nursing, Faculty of Nursing, Tanta University, Egypt \\ *Corresponding Author's Email: saharmelika2020@gmail.com
}

\begin{abstract}
Introduction:Idiopathic Thrombocytopenic Purpura is an autoimmune blood disorder associated with decreased platelets level less than normal, which may result in bleeding occurrence. The actual cause is unknown. It may be progressive if untreated but may remit spontaneously for several years. Aim: The aim of this study was to evaluate the effect of health education programs on nurses' performance for children with Idiopathic Thrombocytopenic Purpura. Methods: The design of the study was a quasi-experimental research design that was used for 50 nurses working at the Pediatric Hematology Unit of Tanta University Hospital and Pediatric Hematology Unit of Al-Mabra Tanta Hospital. Tools: Two Tools were used to collect the required data - a structured interview scheduled to collect biosocial data of nurses and an observational checklist regarding nursing practice. Results: The results of the present study represented that three-quarters of the nurses had poor knowledge and all of them had an unsatisfactory level of practice before the educational program implementation, while immediately and after one month from the program the total scores of nurses' knowledge and practice were improved. Conclusion: There was a significant improvement in nursing knowledge and practice in relation to nursing care for children with Idiopathic Thrombocytopenic Purpura. Recommendations: In-service training program should be conducted periodically for nurses' teaching the basic knowledge and clinical skills.
\end{abstract}

Keywords: Educational Program; Nurses' Performance; Children with-Idiopathic Thrombocytopenic-Purpura

\section{INTRODUCTION}

Idiopathic Thrombocytopenic Purpura (ITP) is one of the most common acquired-platelets disorders in children, it is caused by the development of autoantibodies against platelets which lead to thrombocytopenia, in the absence of other reasons such as medication, infections, cancer, and other autoimmune illnesses. The term Idiopathic refers to the cause is unknown; Thrombocytopenia means a reduction in platelet count in blood; and Purpura refers to purple staining of the skin (Gernsheimer, 2019; Beardsley \& Nathan 2016).

Idiopathic Thrombocytopenic Purpura is divided into two types: acute and chronic. The acute form of ITP is the most prevalent in children between the ages of 2 and 6. Its symptoms usually disappear within six months or few weeks. Chronic kind can occur at any age, with symptoms lasting from six months to several years (Akhtar, Daley \& Nakasato, 2017; (Mantadakis, Farmaki \& Buchanan, 2017). In 2021, the annual global incidence of ITP is expected to be 2.2-5.3 per 100.000 children (Leach et al., 2021; Provan \& Newland, 2017).

In children, the specific aetiology of the disease is unknown. They may be infected with a virus for three weeks before acquiring it. Their immune system generated antibodies to fight the infection, but they made antibodies that may cling to platelet cells by accident. Because these antibodies are recognised by the spleen, which helps the body fight infection, the platelets in the children's system are destroyed, resulting in lower circulating platelet levels than usual. 
Clinical manifestations are easy bruising with petechial, purpura, or ecchymosis, particularly over bony prominence. Bleeding from mucous membranes, such as epistaxis, gums bleeding, and internal hemorrhage with evidence of hematuria, hematemesis, melena, and hematomas over the lower extremities that may result in chronic leg ulcers (Beck et al., 2015; Rodeghiero, Stasi \& Gernsheimer, 2013).

Treatment of ITP includes corticosteroid which is the best choice for initial treatment. Intravenous Immunoglobulin (IVIG) and anti-D immune globulin have also been recommended. These medications are used to elevate platelet count quickly in urgent situations, but they are not recommended for long-term therapy because of limited response and long-term toxicity (Carcao et al., 2015; Provan, Stasi \& Newland, 2018).

A pediatric nurse plays an integral role in caring for those children. The general principles of children's care include the detection of early signs of internal bleeding, remaining children uninjured, increasing their daily activity, remaining free from infection, and recognizing early signs of infection to allow for proper early treatment (Lambert \& Gernsheimer, 2017).

Continuously evaluating safety environment and identifying possible risks, the child has become thoroughly acquainted with the environment, put call light in reach and teach how to use assistance, respond immediately to call light. For the child learning to walk with a helmet, knee and elbow pads are recommended to protect the head from injuries due to frequent falls. It is recommended to play with safe and age-appropriate toys. The home environment should be modified to promote safety (as padding the walls, cover the floor with heavy carpets, pad sharp-edged furniture) (Abadi, Yarchovsky-Dolberg \& Ellis, 2015; Raj, 2017).

Bleeding prevention through using a special toothbrush and avoid hard foods. Adolescents should be advised to avoid using safety razors with blades and encouraged to use electric shavers. The subcutaneous route is substituted for intramuscular injections and vein puncture for blood samples is preferred. The aspirin-containing compound shouldn't be used. Assess the intravenous site for leakage, bleeding, and applying pressure for 15 minutes to puncture sites (Stasi \& Provan, 2017; Godeau, 2014).

Control of blood by application of the RICE technique, as R stands for rest of the limb or portion, I for applying cold packs to promote vaso-constriction, C to suppress or apply pressure for at least 10-15 minutes to the bleeding areas and $\mathrm{E}$ to increase the site affected above the heart level. The nurse suspects when a kid has indications of internal bleeding such as headache, slurred voice, loss of consciousness and black tarry stool. Limit joint involvement and treat hemarthrosis when it occurs and immobilize the joint in a mild flexion posture (Tripathi, Mishra \& Kumar, 2016; Imbach \& Crowther, 2018).

Once bleeding has been controlled, range of motion exercises are performed to strengthen muscles and joints and to prevent flexion contractures. Encourage the child to maintain appropriate weight because excess weight can place added stress on joints. The nurse must use non-pharmacological methods to relieve pain such as distraction, relaxation, application of hot and cold compresses. Provide emotional support to the child and their parents and help them to verbalize their feelings. The nurse must understand and be sensitive to their needs (Reese \& Hauben, 2010; Godeau, 2014).

\section{The Study's Significance}

Idiopathic Thrombocytopenic Purpura is immunemediated destruction of platelets that results in a decrease in platelet count and a higher risk of bleeding. It affects 1.9 to 6.4 children per 100,000 in Egypt each year, and $5-10 \%$ of children with chronic ITP have severe bleeding, making treatment difficult. Thus, improving nurses' knowledge of ITP in children through educational programmes is critical in raising their awareness of the condition and enhancing the survival rate of such children. The study's goal was to see how an educational programme affected nurses' performance in caring for children with Idiopathic Thrombocytopenic Purpura.

\section{Aim}

The aim of this study was to evaluate the effect of educational program on nurses' performance for children with Idiopathic Thrombocytopenic Purpura.

\section{Research Hypothesis}

Nurses' performance for children with Idiopathic Thrombocytopenic Purpura is expected to be improved after an educational program implementation.

\section{Subjects and Method}

Research Design: A quasi-experimental research design was used in this study. Setting: The study was 
conducted at Pediatric Hematology Unit of Tanta University Hospital and Pediatric Hematology Unit of Al- Mabra Tanta Hospital. All nurses (50) who work at the above stated sites comprised thirty-five hospital nurses and fifteen Al-Mabra Hospital nurses.

\section{Tools of Data Collection}

Two tools were used to gather necessary data, they were included the following:

\section{Tool I: Structured Interview Schedule}

The researcher designed the study to evaluate nursing knowledge before, immediately and after one month of implementation of the training programme. There were three parts:

Part 1: Socio-demographic Characteristics of the Studied Nurses such as; socio-demographic features of the nursing staff examined such as: old age, education, years of Pediatric Hematology Unit experience, and participation in relevant training courses.

Part 2: Nurses' knowledge about Idiopathic Thrombocytopenic Purpura Definition, etiology, predisposing factors, pathophysiology, clinical manifestations, complications, treatment and nursing interventions.

Part 3: Nurses' knowledge related to health problems for ITP in children: Bleeding, easily bruising, pain, and high risk for infection due to immunosuppressant drugs and disease process.

Total score of nurses' knowledge was calculated as following:

- $<60 \%$ was considered poor knowledge.

- $60-<75 \%$ was considered fair knowledge.

- $75-100 \%$ was considered good knowledge.

Tool II: Observational Checklist to Assess Nurses' Practice for Children with Idiopathic Thrombocytopenic Purpura

\section{It includes the following items:}

- Maintenance of safe hospital environment

- Prevention of injury (dental care, physical exercise, skin care)

- Control of bleeding episodes

- Administration of medication and blood products

- Monitor growth and development
- Emotional and psychological support

- Discharge planning

- Documentation

\section{Scoring System for Nurses' Practice was as Follow:}

- Done correctly and complete was scored (1)

- Done incorrectly or not done was scored (0)

\section{METHODOLOGY}

1. An official permission to conduct this study was obtained from the Faculty of Nursing, Tanta University directed to administrators of Pediatric Hematology Unit at Tanta University Hospital and Al- Mabra Tanta Hospital to obtain their approval and cooperation for carrying out this study.

2. Ethical and legal considerations: The information obtained from the nurses was informed of confidentiality. The nature of the study did not harm the whole sample. Oral consent of the nurses was obtained to participate in the study after the researcher explained its aim and benefits. Nurses who accepted to take part in the current study were engaged and had the right at all times to withdraw from the study.

3. Two tools were used for gathering data.

4. Reliability of the developed tool was tested through internal consistency. The value of Cronbach's alpha coefficient was $=0.949$.

5. Content validity: The study tools were submitted to a panel of five pediatric nursing experts in order to check the validity of the content and clarity of the questionnaire. Changes have been made accordingly. After calculating the validity index content of the items, the questionnaire was calculated on the basis of expert opinions and was 99.1 per cent.

6. A pilot study: Five sample nurses $(10 \%)$ conducted a pilot study to test the tool for clarity, application, feasibilities and required modifications. The pilot sample was not included in the study.

7. Study phases: Four phases of the study were conducted:

I. Assessment phase: For all study subjects, the researcher performed a baseline study and evaluated nursing knowledge of children with Idiopathic Thrombocytopenic Purpura's health requirements before, immediately and within a month of application. (Tool I). 
The researcher evaluated the practice of the actual nurses before, immediately and within one month. Alternatively, the researcher was available 2 days a week in the above-mentioned settings as all studied nurses were observed in all morning shifts while taking care of children with ITP.

\section{Planning phase:}

- Setting educational program objectives

- Preparing the content covered by the reasons behind the session's implementation

\section{Implementation phase:}

- The education program for nurses was carried out by following sessions in accordance with the evaluation of actual needs of nurses.

- Education was carried out in six sessions in two weeks. The duration of each session was around 30-45 minutes including discussion periods based on progress and feedback from nurses.

- Various teaching methods have been used including lectures, group discussions and demonstrations to promote the understanding of nurses.

- The nurses studied were divided into small groups, with five nurses in each group.

- The training programme for each group was conducted separately through successive sessions according to the real need evaluation of the studied nurses.

- Each session began with a summary of what the previous session had discussed.

\section{The Sessions Dealt with the following Subjects:}

First Session: The first session was concerned with the development of bleeding disorders, definition of types, epidemiology, etiology, predisposing factors, Idiopathic Thrombocytopenic Purpura pathophysiology.

Second session: Idiopathic Thrombocytopenic Purpura has been focused on clinical manifestations, health problems, diagnosis, treatment and complicated conditions.

Third session: The focus was on bleeding prevention through the maintenance of a safe hospital environment, injury prevention through dental care and exercise.

Fourth Session: It focussed on nursing interventions for episodes of bleeding, medication and blood transfusion administration. The questions of the nurses were answered by the end of the session.

Fifth Session: Methods for monitoring child development and growth, skincare, blood removal and pain management.

Sixth Session: The focus was on the required health education for Idiopathic Thrombocytopenic Purpura and discharge plan parents and their children. In the context of discussion of related concepts and demonstrations of health education, the contents presented to nurses were simulated on children and parents. Each session ended with a summary of its contents and feedback from the nurses to ensure the maximum benefit to the nurses.

IV. Evaluation phase: Evaluation of the knowledge and practice of nurses by the use of the same evaluation tools has been conducted. Each nurse was assessed immediately after the programme was implemented (after the test) and one month later, and the pre-test levels were compared. Data were gathered for one year between December 2019 and November 2020. The study lasted for 2 years.

\section{RESULTS}

\begin{tabular}{|c|c|c|}
\hline \multirow{2}{*}{$\begin{array}{l}\text { Socio-demographic } \\
\text { Characteristics of Nurses }\end{array}$} & \multicolumn{2}{|c|}{ The Studied Nurses $(n=50)$} \\
\hline & No. & $\%$ \\
\hline \multicolumn{3}{|l|}{ Age in Years } \\
\hline \multirow{4}{*}{$\begin{array}{l}20-<30 \\
30-<40 \\
\quad \geq 40 \\
\text { Range } \\
\text { Mean } \pm \text { SD }\end{array}$} & 11 & 22.0 \\
\hline & 12 & 24.0 \\
\hline & 27 & 54.0 \\
\hline & \multicolumn{2}{|c|}{$\begin{array}{c}20-59 \\
40.340 \pm 11.605\end{array}$} \\
\hline \multicolumn{3}{|c|}{ Sex } \\
\hline Male & 1 & 2.0 \\
\hline Female & 49 & 98.0 \\
\hline \multicolumn{3}{|c|}{ Educational Level } \\
\hline $\begin{array}{l}\text { Secondary Nursing school } \\
\text { Health Technical Institute } \\
\text { Bachelor of Nursing } \\
\text { Science }\end{array}$ & $\begin{array}{c}32 \\
12 \\
6\end{array}$ & $\begin{array}{l}64.0 \\
24.0 \\
12.0\end{array}$ \\
\hline \multicolumn{3}{|c|}{ Years of Experience } \\
\hline$<10$ & 15 & 30.0 \\
\hline $10-<20$ & 11 & 22.0 \\
\hline $20-<30$ & 12 & 24.0 \\
\hline$\geq 30$ & 12 & 24.0 \\
\hline Range & \multirow{2}{*}{\multicolumn{2}{|c|}{$\begin{array}{c}1-41 \\
19.540 \pm 11.780\end{array}$}} \\
\hline Mean \pm SD & & \\
\hline \multicolumn{3}{|c|}{ Attendance of any Training Programs about ITP } \\
\hline Yes & 0 & 0.0 \\
\hline No & 50 & 100.0 \\
\hline
\end{tabular}


Table 1 illustrates the socio-demographic characteristics of the studied nurses. It was observed that slightly more than half (54\%) of the studied nurses' age was 40 years and above with mean age \pm SD (40.3 \pm 11.6$)$. Regarding their sex, it was clear that the majority $(98 \%)$ of nurses were female. The educational level of about two thirds (64\%) of the nurses were secondary nursing school while near to one quarter (24\%) of them had a technical nursing institute. The rest $(12 \%)$ of them had Bachelor of Nursing Science.

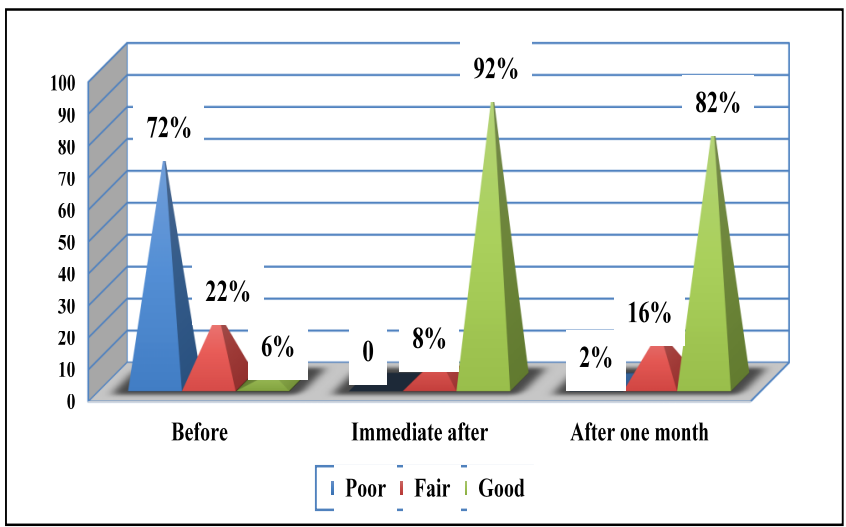

Figure 1: Total Scores of Nurses'-Knowledge about Idiopathic-Thrombocytopenic-Purpura
Figure 1 represents the total scores of nurses knowledge about ITP before, immediately and after one month from the educational program implementation. It was found that before the educational program the total scores for nearly three quarter $(72 \%)$ of nurses were poor. While immediately and after one month from the program implementation, the majority $(92 \%)$ of them and the most $(82 \%)$ of them had good scores respectively with highly statistically significant difference before, immediately and after one month from the educational program $(P=0.0001)$.

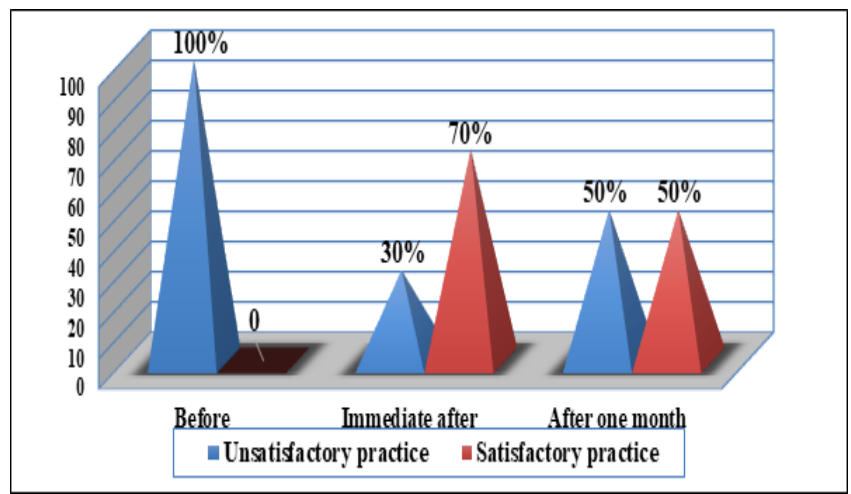

Figure 2: Total Scores of Nurses' Practice for Children with Idiopathic Thrombocytopenic Purpura

Table 2: Correlation between Mean of Total Nurses' Knowledge and Practice Scores and Their Socio-Demographic Data

\begin{tabular}{|c|c|c|c|c|c|c|c|c|c|c|c|c|}
\hline \multirow{3}{*}{$\begin{array}{c}\text { Socio- } \\
\text { demographic data }\end{array}$} & \multicolumn{6}{|c|}{ Total knowledge scores $(n=50)$} & \multicolumn{6}{|c|}{ Total practice scores $(n=50)$} \\
\hline & \multicolumn{2}{|c|}{ Before } & \multicolumn{2}{|c|}{ Immediate after } & \multicolumn{2}{|c|}{ One month after } & \multicolumn{2}{|c|}{ Before } & \multicolumn{2}{|c|}{ Immediate after } & \multicolumn{2}{|c|}{ One month after } \\
\hline & $r$ & $P$ & $r$ & $P$ & $r$ & $P$ & $r$ & $P$ & $r$ & $P$ & $r$ & $P$ \\
\hline Age in years: & -0.361 & $0.010 *$ & -0.402 & $0.004 *$ & -0.131 & 0.365 & -0.422 & $0.002 *$ & -0.940 & 0.0001* & -0.692 & $0.0001 *$ \\
\hline$\cdot \operatorname{Sex}$ & -0.161 & 0.265 & -0.042 & 0.771 & 0.064 & 0.654 & -0.429 & $0.002 *$ & -0.094 & 0.518 & -0.132 & 0.361 \\
\hline -Educational level: & 0.758 & $0.0001^{*}$ & 0.181 & 0.210 & 0.137 & 0.343 & 0.136 & 0.346 & 0.401 & $0.001^{*}$ & 0.524 & $0.0001 *$ \\
\hline $\begin{array}{l}\text { - Years of } \\
\text { experience }\end{array}$ & 0.360 & $0.010^{*}$ & 0.385 & $0.006 *$ & 0.117 & 0.417 & 0.376 & $0.007 *$ & 0.688 & $0.0001 *$ & 0.676 & $0.0001^{*}$ \\
\hline
\end{tabular}

Figure 2 demonstrates the total scores of nurses' practices provided to children with ITP before, immediately and after one month from the program implementation. It was apparent that before the program all nurses had unsatisfactory level of practice while as immediate and one month after $70 \%$, and $50 \%$ had satisfactory level of practice respectively.

Table 2 presents the correlation between the total nurses' knowledge, and practice scores, and their sociodemographic data before, immediate after, and after one month from the educational program implementation. It was cleared that there was a negative correlation between the nurses' age and their knowledge and practice. On the other hand, a positive correlation was detected among the nurses' educational level and years of experience with their knowledge and practice. Nurses displaying a high level of education with more years of experience had good knowledge and practice before, immediately and one month after program implementation. 


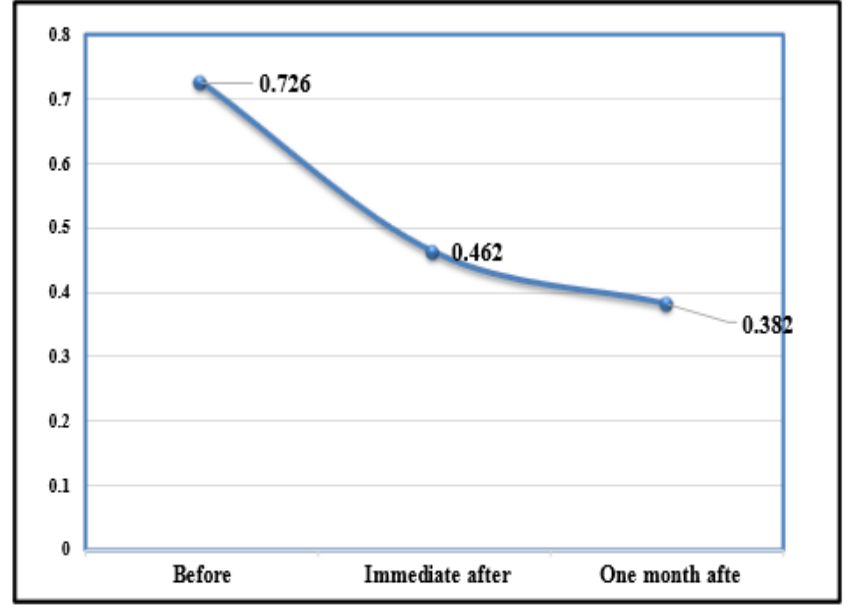

Figure 3: Correlation between Total Scores of Nurses' Knowledge and Practices for Children with ITP

Figure 3 presents the correlation between total scores of nurses' knowledge and practice for children with ITP before, immediate, and after one month of program implementation. It was observed that there was a positive correlation between nurse's knowledge and practice before, immediately, and one month after program implementation.

\section{DISCUSSION}

Idiopathic Thrombocytopenic Purpura (ITP) is a serious disease that affects many aspects of the affected child's life. This disease leads to bleeding such as nosebleeds, bleeding from gums, easy bruising, hematemesis, hemoptysis, hematuria, and melena. In addition to, intracranial hemorrhage, which is a lifethreatening condition and most often occurs within the first 12 months following diagnosis (Tripathi, Mishra \& Kumar, 2016; Imbach \& Crowther, 2018).

Health problems can prevent the child from practicing his/her normal life as all children within his/her age. There is no doubt that upgrading the nurses' awareness about ITP is essential to improve an early diagnosis and treatment of this unpredictable and misunderstood disease. Continuous educational program for nurses keeps them up to date on the latest advances in nursing care provided to those children, so the present study was conducted to evaluate the effect of educational program on nurses' performance for children with Idiopathic Thrombocytopenic Purpura (Reese \& Hauben, 2010).

Regarding the studied nurses' educational level, the present study revealed that slightly less than two-thirds of them are graduated from secondary nursing school while the least of them had completed their university nursing education. Nurses' educational level is an important factor which has a direct effect on the quality of care given to these children, especially children with blood diseases because they are always in a serious condition and need advanced and specialized care (Abadi, Yarchovsky-Dolberg \& Ellis, 2015).

Yousif (2014) was in the same line with the current study as he revealed that more than half of the studied nurses graduated from secondary nursing school (Yousif, 2014). Oday (2017) disagreed with these results and stated that about two-thirds of them had a bachelor's degree in nursing. Besides Vitoria (2018) who mentioned that the majority of the studied nurses had university nursing education and the least of them had hematology nursing specialization (diploma degree) and master's degree, also he mentioned that hematology nurses should attend an accredited university or college and graduate with a Bachelor of Science in Nursing degree.

With respect to the overall knowledge of Idiopathic Thrombocytopenic Purpura, about 3/4 of the nurses had low levels of knowledge before the programme of education. This may be ascribed to just about two-thirds of them being finished in secondary school and they have not all taken ITP-related training courses. In order to acquire the necessary information, when necessary, the invariability of books particularly in this field helped (Abrams, 2014). In line with the results presented, Rosenthal (2017) revealed that most nurses had inadequate understanding and over half of them had poor ITP knowledge prior to the programme's implementation.

On the opposite, immediately after the program application, nurses' knowledge improved and the majority of them obtained good scores. This could be attributed to the content of the programme which was developed based on the nurses' needs, its clarity and simplicity, use of audiovisual aids, availability of the researcher in the field for more clarification, using simple language, and frequent repetition to fix the knowledge. Suchitra \& Lakshmi (2019) were in accordance with the current study as they reported that education has a positive impact on retention of knowledge and attains competency in all categories of staff.

Furthermore, one month after the program 
application, this percentage was slightly reduced and most of them had good scores in all the items of knowledge. This indicated that the improvement in knowledge was partially lost one month after the application of the guidelines. This result might be explained by the fact that knowledge retention is usually affected by time (Wahlheim, Maddox \& Jacoby 2014). Morehead \& Rhodes (2016) were in harmony with the current study as they confirm that information can be easily forgotten if they are not refreshed periodically.

In relation to the nurses' level of practice for children with ITP, the present study showed that all nurses had an unsatisfactory level of practice before the educational program. This might be related to the fact that all of them were not receiving any previous training program about ITP, near two-thirds of them graduated from secondary nursing school, years of experience for third of them less than ten years, absence of motivation from the administration, and no pre-employment orientation program.

Abd El-Naeem (2015) agreed with the present study and stated that the majority of the studied nurses had a poor level of practice before the implementation of an educational program, due to the absence of preemployment orientation, deficiencies in training courses, and increased workload which may prevent them from the reading to upgrade their practice. On contrary, immediate after the program implementation, the present study showed that there was a statistically significant improvement in nurses' practice. This may be due to the effectiveness of the education program. Abdelmonem \& Abd El-Aziz (2019) have agreed to the present conclusion that the nurses' practices in the haematology department have improved significantly after the training module.

Furthermore, the overall number of nurses' practices has been significantly decreased after a month of programme implementation. This showed that improved practice after a month of programme implementation dropped somewhat, but better than its results immediately before implementation. This may be because nurses need to retake their education programmes regularly to maintain their acceptable level of practice.

Potter \& Perry (2016) were consistent with the current research and stated that the results of nurse practice were marginally decreased after three months since the majority of nurses were satisfied in all praxis items. Aiken (2014) also said that nursing abilities should be enhanced through training, ongoing education, and professional conferences. It offers children excellent, efficient healthcare and also needs to enhance their nursing abilities to keep pace with new methods and treatment.

Concerning correlation between the total nurses' knowledge, practice, and their socio-demographic characteristics. It was cleared that there was a negative correlation between nurses' age, knowledge, and practice. This finding may be explained in the light of the fact that young nurses have fresh knowledge and still remember what they had studied in their academic institution, unlike older nurses. Furthermore, young nurses are usually active than older nurses; they have good physical fitness and are creative in achieving the nursing practice. On the other hand, nurses by their mid40 years of age start to base their practice on routine care more than up-to-date knowledge, also experience sensory limitation related to vision and hearing acuity, in addition to risks of contracting chronic illness and weight gain.

Robinson (2016) was congruent with the present study as he reported that older nurses have less access to continuing professional development activities when compared to their younger colleagues. On the other hand, Amatya \& Gorkhali (2015) were incongruent with the present study as they stated that the number of years in a job in the same or similar situations increases the level of knowledge and practice of this field and may create competence.

Moreover, the present study revealed that there was a statistically significant difference between scores of total nurses' knowledge, practice, and their education before, immediate, and after one month from the program implementation. Also, a positive correlation was detected among nurse's educational level with their knowledge and practice. This may be due to the highly qualified nurses who have bachelor's degree able to provide special care and they usually have the right knowledge and skills to meet the needs of these children more than nurses who have secondary nursing school.

Abolwafa \& Mohamed (2016) were in agreement with the current study as they stated that the highest knowledge and practice scores were found among nurses having bachelor's degree in nursing science which provide them with more information. Also, continuing education and advancement of nurses 
increase the knowledge, the opportunity for a shared experience with colleagues, and provide support for professional decision making and action.

Correlation between nurses' knowledge and practice regarding care of ITP children before, immediate, and one month after the educational program the current study represents that there was a positive correlation between nurses' knowledge and practice. This may be due to the fact that the theoretical part of the program was effective in modulating nurses' performance as the nurses acquire new knowledge and skills become able to perform it perfectly during their practice. So, knowledge should be accompanied by the competency level of practice.

Saillour \& Glenisson (2016) were in harmony with the present finding and revealed that nurses should maintain a high level of nursing knowledge and practice to be effective in practice and if they work with inadequate knowledge, their practice will be incomplete and inadequate. Also, George (2017) mentioned that nursing education and practice have an interdependent relationship, each stimulates and reflects the other's progress, and each is affected by changes in the other, and that may reflect the importance of integration between theory and practice.

\section{CONCLUSION}

There was a significant improvement in nursing knowledge and practice in relation to nursing care for children with Idiopathic Thrombocytopenic Purpura. The study will help to develop an assessment system for nurses on current methods to enhance their knowledge and practice.

\section{Recommendations}

The following suggestions were made on the basis of the results of the current study:

1. In-service training programme for the teaching of fundamental medical skills such as prevention and control bleeding for ITP children should be performed frequently and regularly.

2. To encourage nurses to participate in training programmes that should be performed in the workplace, an accreditation and certification system should be established.

3. Special training sessions on monitoring children's growth and development and the significance of handwashing should be developed and given to all haematological nurses.

\section{Conflict of Interests}

The authors declare that they have no conflict of interests.

\section{ACKNOWLEDGEMENT}

The authors are thankful to the institutional authority for completion of the work.

\section{REFERENCES}

Abadi, U., Yarchovsky-Dolberg, O., \& Ellis, M. (2015). Immune thrombocytopenia: recent progress in pathophysiology and treatment. Clinical and Applied Thrombosis/Hemostasis, 21(1), 397-404.

Abd El-Naeem, M. (2015). Identifying the effect of a Basic Life Support teaching program on nurses' knowledge and skills at emergency care unit. Assiut Scientific Nursing Journal, 6(3),15-25.

Abdelmonem, M., \& Abd El-Aziz, F. (2019). Study the significance of educational program on the nursing process. The International Conference on Developing the Higher Education in Egypt, 13(3), 13-8.

Abolwafa, N., \& Mohamed, A. (2016). Effect of educational training about cardiopulmonary resuscitation on pediatric nurses' practice. International Journal of Current Research, 9(6), 8781-8788.

Abrams, C. (2014). Low blood platelets: Goldman's Cecil Medicine (24 ${ }^{\text {th }}$ ed.). Elsevier Saunders Co.

Aiken, L. (2014). Education policy initiatives to address the nurse shortage in the United States. Health Affairs, 28(4), 646-656.

Akhtar, A., Daley, M., \& Nakasato, C. (2017). The risk of Immune Thrombocytopenic Purpura after vaccination in children and adolescents. American Journal of Pediatrics, 129(2), 248-255. 
Amatya, M, \& Gorkhali, B. (2015). Knowledge amongst nepalese health personnel: Janaki Medical College. International Journal of Medical Sciences, 3(1):25-30.

Beardsley, D., \& Nathan, G. (2016). Platelets Abnormalities in Infancy and Childhood ( $7^{\text {th }}$ ed.). WB Saunders Co.

Beck, C., Nathan, P., Parkin, P., Blanchette, V., \& Macarthur, C. (2015). Corticosteroids versus intravenous immune globulin for the treatment of acute immune thrombocytopenic purpura in children: A systematic review and metaanalysis of randomized controlled trials. Journal of Pediatrics, 147(4), 521-527.

Carcao. M., Zipursky, A., Butchart, S., Leaker, M., \& Blanchette, V. (2015). Short-course oral prednisone therapy in children presenting with acute immune thrombocytopenic purpura. Acta Paediatrica, 42(4), 71-74.

George, N. (2017). Idiopathic Thrombocytopenic Purpura: A Practice guideline developed by explicit methods. Blood Review, 88(12), 24-29.

Gernsheimer, T. (2019). Definitions and outcome criteria in immune thrombocytopenic purpura of children: report from an international working group. Blood, 113(7), 2386-2392.

Godeau, B. (2014). Immune thrombocytopenic purpura: major progress in knowledge of the pathophysiology and the therapeutic strategy, but still a lot of issues. La Presse Médicale, 43(4), 47-48.

Imbach, P., \& Crowther, M. (2018). Thrombopoietin-receptor agonists for primary immune thrombocytopenia. New England Journal of Medicine, 365(8), 734-741.

Lambert, M., \& Gernsheimer, T. (2017). Clinical updates in adult immune thrombocytopenia. Blood, 129(21), 28292835.

Leach, C., Hay, J., Myron, J., Mark, A., \& Maya, B. (2021). Current Diagnosis \& Treatment: Pediatrics (25 ${ }^{\text {th }}$ ed.). McGraw Hill Co.

Mantadakis, E., Farmaki, E., \& Buchanan, G. (2017). Thrombocytopenic purpura after Measles-Mumps-Rubella vaccination: A systematic review of the literature and guidance for management. Journal of Pediatrics, 156(4), 623-628.

Morehead, K., \& Rhodes, M. (2016). Instructor and student knowledge of study strategies. Journal of Memory and Language, 24(5), 257-271.

Oday, F. (2017). Assessment nurses' practices about hemophilia for children attending heredity blood disease center in Al-Nasiriya City. American Journal of Nursing, 6(3), 345-347.

Potter, P., \& Perry, A. (2016). Basic Nursing: Essentials for Practice. (15 ${ }^{\text {th }}$ ed.). Mosby Co.

Provan, D, \& Newland, A. (2017). International consensus report on the investigation and management of primary immune thrombocytopenia. American Journal of Blood Research, 9(3), 115-128.

Provan, D., Stasi, R., \& Newland, A. (2018). International consensus report on the investigation and management of primary immune thrombocytopenia. Blood, 115(1), 68-186.

Raj, B. (2017). Immune thrombocytopenia: pathogenesis and treatment approaches. Hematology, Transfusion and Cell Therapy, 5(1), 1056-1065.

Reese, J., \& Hauben, M. (2013). Identifying drugs that cause thrombocytopenia: an analysis using three distinct methods. Blood, 116(8), 2127-2133.

Robinson, J. (2016). Evaluation of educational Plan: Meeting the needs of nurses? Journal of Nutritional Sciences and Dietetics, 22(2), 65-69.

Rodeghiero, F., Stasi, R., \& Gernsheimer, T. (2013). Standardization of terminology, definitions and outcome criteria in immune thrombocytopenic purpura of adults and children: Report from an international working group. Blood, 
113(11), 2386-2393.

Rosenthal, M. (2017). The effect of in-service education program of nurse's knowledge. American Society of Hematology Education Program, 20(4), 851-857.

Saillour, F., \& Glenisson, S. (2016). Factors associated with hematology nurses' poor knowledge and practice of transfusion safety procedure in Aquitaine and France. International Journal of Quantum Chemistry, 14(2), 2532.

Stasi, R., \& Provan, D. (2017). Management of immune thrombocytopenia purpura. Mayo Clinic Proceedings, 79(4), 504-522.

Suchitra, J., \& Lakshmi, N. (2019). Impact of education on knowledge, attitudes and practices among various categories of health care workers. Indian Journal of Medical Microbiology, 25(3), 181-187.

Tripathi, A., Mishra, S., \& Kumar, A. (2016). Megakaryocyte morphology and its impact in predicting response to steroid in immune thrombocytopenia. Platelets, 25(7), 526-531.

Vitoria, N. (2018). Nurses knowledge of hematology clinics of on systemization nursing care. American Journal of Nursing, 19(4), 1658-1660.

Wahlheim, N., Maddox, G., \& Jacoby, L. (2014). The role of reminding in the effects of spaced repetitions on cued recall: Sufficient but not necessary. Journal of Experimental Psychology: Learning, Memory, and Cognition, 40(8), 94-105.

Yousif, A. (2014). The perspective of the pediatric nurses toward hemophilia in the teaching hospitals of Kirkuk city. Kufa Journal for Nursing Sciences, 4(3), 203-206. 